Open Access

\title{
Unusual localization of a primary pleomorphic malignant fibrous histiocytoma on the mitral valve: a case report and review of the literature
}

\author{
Edvin Prifti ${ }^{1}$, Fadil Ademaj ${ }^{2,3^{*}}$, Majlinda Ikonomi $^{1}$ and Aurel Demiraj ${ }^{2}$
}

\begin{abstract}
Introduction: It has been reported that cardiac malignant fibrous histiocytomas occur more frequently in the left side of the heart, especially in the left atrium, but rarely invade the mitral valve. We present a case with a giant malignant fibrous histiocytoma with an unusual localization involving almost the entire left atrium, mitral valve, and left superior pulmonary vein.

Case presentation: We describe the case of a 54-year-old woman from Kosovo admitted to our emergency department with dyspnea. A transthoracic echocardiography demonstrated a giant mass localized on her left atrium. Our patient underwent emergent total surgical removal of the mass. The mass extended between her left superior pulmonary vein, and extended to her left atrium and the posterior mitral valve leaflet. We formulated a surgical plan for total separation of the mass from the endocardium. Total removal was performed and her left side pulmonary veins were entirely freed from the mass. We then performed a mitral valve replacement. The differential diagnosis included other masses of the left atrium, including thrombi, vegetations, and cardiac tumors. Postsurgical histopathologic results showed a pleomorphic malignant fibrous histiocytoma. Six monthly follow-up cardiac and abdominal sonographic examinations revealed no tumor recidivism.
\end{abstract}

Conclusion: We reviewed 90 cases with malignant fibrous histiocytoma reported in the literature. Our case was especially unusual because of the primary location in the mitral valve, the pleomorphic variant, and the dimensions and extension. Complete surgical resection is mandatory to ameliorate symptoms and to obtain histologic information.

Keywords: Left atrium, Mitral valve, Pleomorphic malignant fibrous histiocytoma

\section{Introduction}

The first reported case of a cardiac malignant fibrous histiocytoma (MFH) was published by O'Brien et al. [1] in 1964 and the first case undergoing surgical removal in 1978 [2]. In 2001, Okamoto et al. [3] analyzed 46 cases of reported MFH. Since then, 44 additional cases of MFH have been reported, resulting in a total of 90 cases of MFH. Cardiac MFH occur more frequently in the left side of the heart, especially in the left atrium [4-6], but rarely invade the mitral valve. The pleomorphic variant of MFH is rarely found and comprises a high cellularity with bizarre tumor cells with marked atypia and high mitotic index. Here, we report the case of a patient with a pleomorphic MFH invading her mitral valve that underwent successful surgical removal. To the best of our knowledge, this is the first reported case of a pleomorphic variant of such dimensions and tumor extension involving the mitral valve.

\footnotetext{
* Correspondence: fadilademaj1971@gmail.com

${ }^{2}$ Division of Cardiology, Regional Hospital of Gjakovo, Gjakovo, Kosovo

${ }^{3}$ Division of Heart Disease, Gjakovo Hospital, Rr. Prizren, Gjakove, Kosove

Full list of author information is available at the end of the article
}

\section{Case presentation}

A 54-year-old woman from Kosovo was admitted to our hospital with dyspnea. On clinical examination, our 
patient's blood pressure was $100 / 60 \mathrm{mmHg}$, and her pulse rate was 130 beats per minute with a regular rate and rhythm. Cardiac auscultation revealed a diastolic murmur. End-inspiratory crackles suggested pulmonary edema. Two-dimensional transthoracic echocardiography revealed a giant mass originating from her posterior mitral valve leaflet, occupying almost her entire left atrial cavity. Cerebral, thoracic, and abdominal computed tomography was also performed, showing no evidence of additional tumors.

Our patient underwent emergency surgical removal of the cardiac tumor. Intraoperative transesophageal echocardiography was performed, which confirmed the presence of the tumor (Fig. 1a). Our patient underwent bicaval cannulation. Her aorta was clamped and anterograde cardioplegia was administered. Then her left atrium was opened at the interatrial groove. A giant yellowish-white tumoral mass was identified, occupying almost her entire left atrial cavity (Fig. 1b). The mass had invaded her left superior pulmonary vein and extended into the posterior aspect of her left atrium. The tumor invaded the posterior leaflet of her mitral valve. The tumor mass was carefully detached from the endocardium (Fig. 1c) and then entirely removed, including the posterior leaflet of her mitral valve, which was replaced with a $29-\mathrm{mm}$ St Jude mechanical prosthesis (Fig. 1d).

Macroscopically, the excised lesion was composed of multiple irregular soft tissue fragments (Fig. 2). After the surgical excision, the mass was fixed in formalin, paraffin embedded, sectioned at 3- $\mu \mathrm{m}$ thick, and stained conventionally with hematoxylin and eosin. Examination of the histology revealed a high grade sarcoma composed of a fusicellular proliferation in a partial storiform pattern, with irregular fascicles, high cellularity, and pleomorphic and bizarre tumor cells with marked atypia and a high mitotic index (Fig. 3a). There were also large areas of necrosis. Immunohistochemical examination results were $25 \%$ positive for Ki-67 in the tumor (Fig. 3b); negative for the muscle markers and the melanocytic markers CD45 and S100 (Fig. 3c); and positive for CD68,

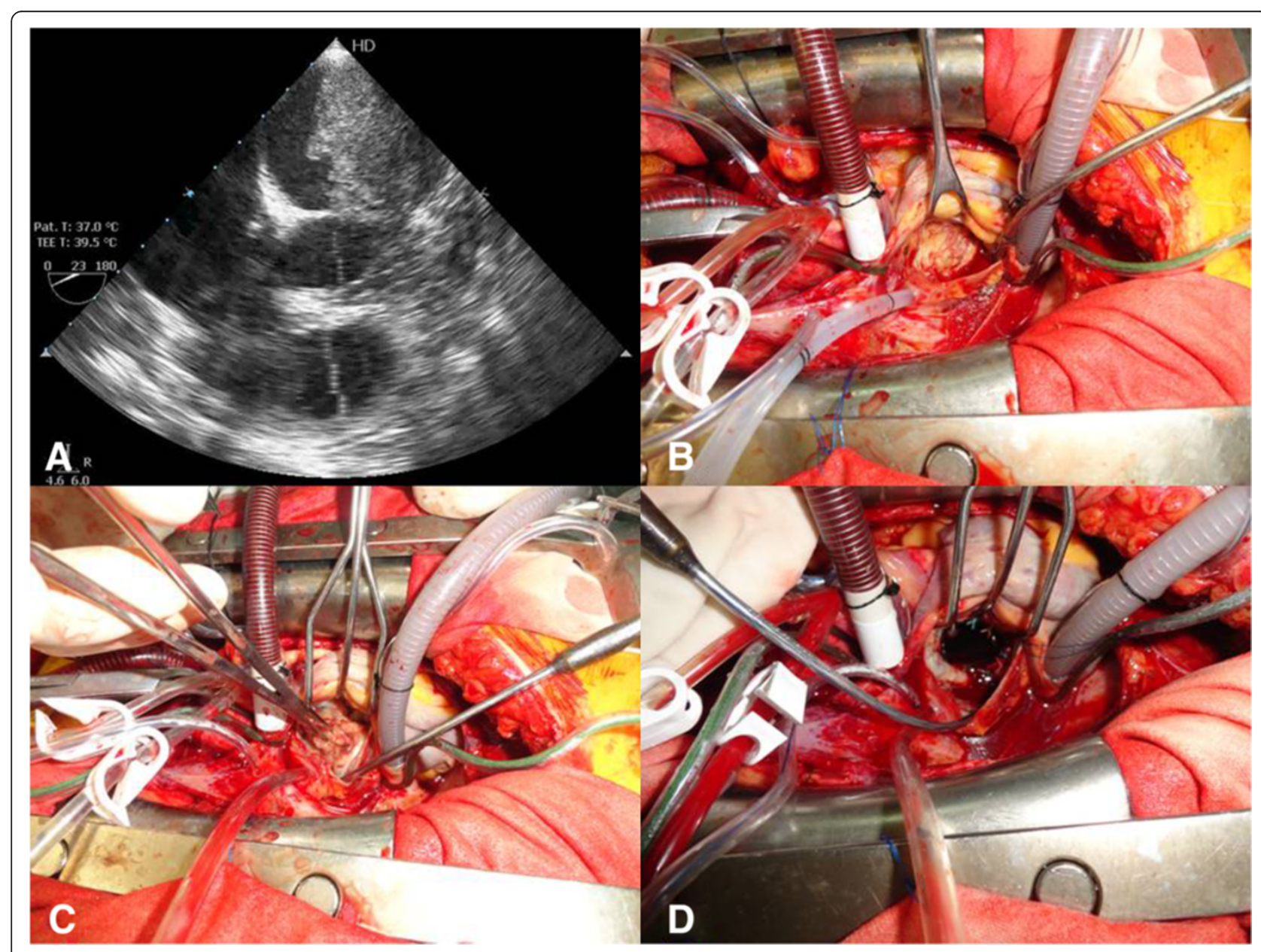

Fig. 1 a Intraoperative transesophageal echocardiography. b Yellowish-white mass in the left atrium. c Removal of the mass from the endocardium. d Mitral valve replacement with a mechanical prosthesis 


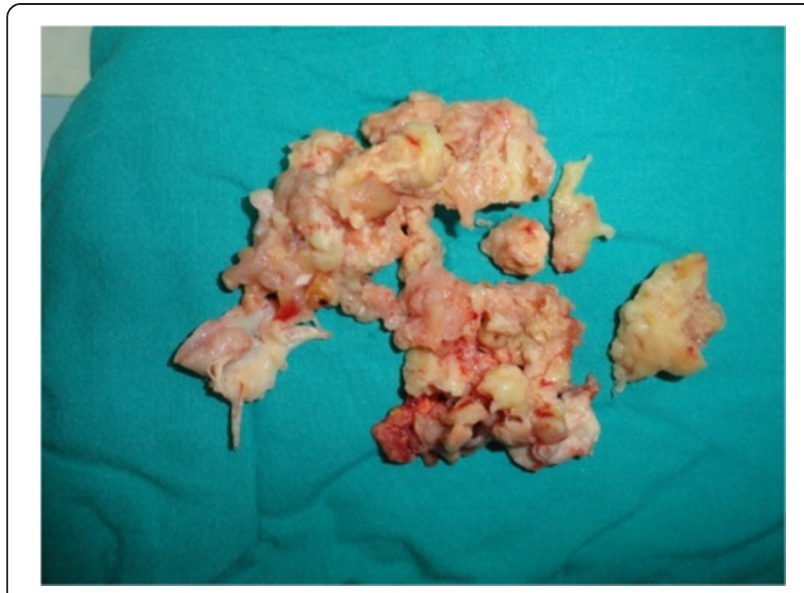

Fig. 2 The excised macroscopic tumoral fragments vimentin, and alpha-1-antitrypsin (Fig. 3d). A diagnosis of pleomorphic MFH was made.

Our patient's postoperative course was uneventful. Six weeks after surgery, she started a six-course chemotherapy regimen of $1.5 \mathrm{mg} / \mathrm{m}^{2}$ of ifosfamide on days 1 to 3 and 80 $\mathrm{mg} / \mathrm{m}^{2}$ of epidoxorubicin on day 1 . The treatment was well tolerated with no unacceptable toxicities. Our patient was still alive with no signs of metastasis six months later.

\section{Discussion}

Primary MFH is the second most common primary cardiac sarcoma, with an estimated incidence of $\sim 1.7 \%$ of cases. It usually affects people of ages 14 to 77 years [3]. After a careful review of the literature, we found reports of a total of 90 cases of primary MFH (Table 1). Primary $\mathrm{MFH}$ is common in the left heart, and it frequently

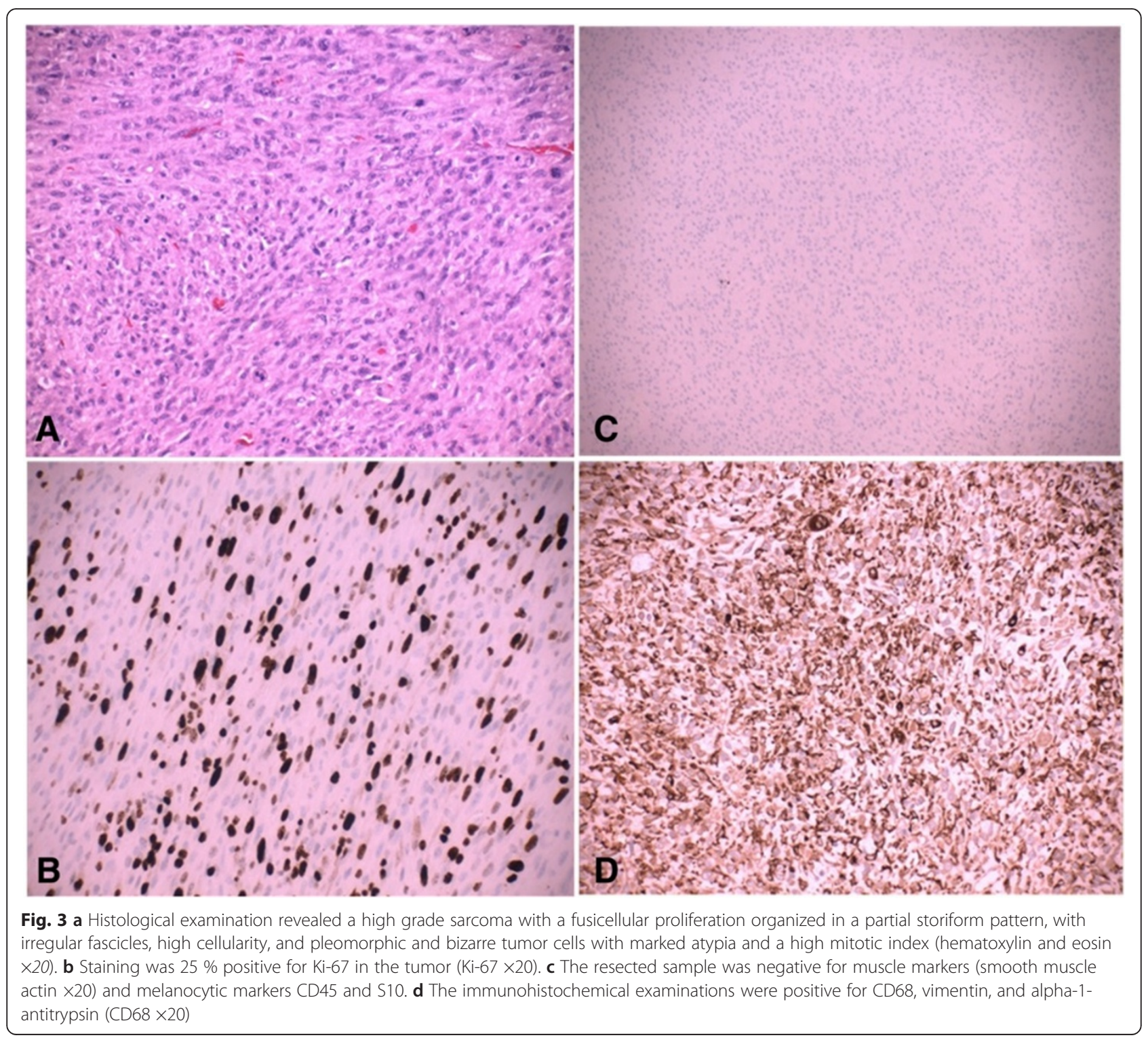


Table 1 Cases reported in the literature with primary cardiac malignant fibrous histiocytoma

\begin{tabular}{ll}
\hline Variables & \\
\hline Total & 60 \\
Female & $14-80$ \\
Age (years) & \\
Localization & 49 \\
Left atrium & 5 \\
Pericardium & 2 \\
Pulmonary veins & 8 \\
Mitral valve & 8 \\
Right ventricle & 9 \\
Right atrium & 5 \\
Left ventricle & 3 \\
Pulmonary artery & 1 \\
Inferior vena cava & \\
Symptoms & $63(70 \%)$ \\
Dyspnea & $40(45 \%)$ \\
Palpitations & $12(13 \%)$ \\
Pleomorphic &
\end{tabular}

manifests as an embolism. Almost $65.5 \%$ of the reported patients presented with a primary MFH in the left atrium. The tumor can also localize in different portions of the left atrium, such as the pulmonary veins [4], septum, and posterior wall. However, the MFH invaded the mitral valve in only eight of the 90 cases [7-13], including our own. In our case, the tumor occupied almost her entire left atrial cavity. Our report seems to be the first reported case with such large tumor dimensions and extension involving the pulmonary vein, left atrium, and mitral valve. The non-septal origin of the mass strongly supports the suspicion of sarcoma. Multiple attachment sites and infiltration of the mitral valve and the atrial and ventricular walls are also indicative of malignancy.

Clinical manifestations of primary MFH are dependent on their size and location. With small tumors, no clinical manifestations are evident. As the tumor grows, the most frequent symptoms are shortness of breath, palpitation, or chest discomfort. The MFH in our case is, to the best of our knowledge, the largest reported.

Histologically, MFH is a variously shaped and multilobulated mass, sessile or pedunculated. It is a tumor of the fibroblasts, with giant cells and atypical nuclear and cytoplasmic findings. The term MFH has now become synonymous of undifferentiated high-grade pleomorphic sarcoma, because the fibrohistiocytic differentiation is not characteristic of a specific tumor type. Sarcomas of myoblastic or fibroblastic origin, including leiomyosarcoma, fibrosarcoma, myxoid fibrosarcoma, and pleomorphic sarcoma (MFH), occur most often as endocardium-based pathologies. The differential diagnosis of cardiac sarcoma in these circumstances is cardiac myxoma, especially in the case of a left atrial intimal-type sarcoma. MFH is a pleomorphic sarcoma composed of fibroblasts, myofibroblasts, and histiocyte-like cells. The pleomorphic variant of the MFH is rarely reported and only had mitral valve involvement in two of the 90 cases we found in the literature $[8,9]$.

The treatment plan for MFH depends on the tumor status, size, histology, location, and metastatic spread. Complete surgical resection is mandatory to ameliorate symptoms and to obtain histologic information. In our case, once we had started surgery, we suspected that we had a malignant tumor. However, owing to the fact that the tumor had invaded her posterior mitral valve leaflet, causing severe mitral valve obstruction, we had to remove her mitral valve as this was the only measure to complete total tumoral mass removal and relieve the mitral valve stenosis.

Although the prognosis of MFH is poor and the possibility of local recurrence and metastasis is high, patients benefit from surgery. Multiple studies have reported a median survival of six months for right-sided tumors, whereas left heart tumors seem to have a better prognosis [14]. The role of chemotherapy and radiotherapy in the treatment of primary cardiac sarcomas has not proven to be beneficial, and complete surgical excision is the only mode of therapy that has been shown to prolong survival.

\section{Conclusion}

Our case was especially unusual because of the primary location of an MFH in the mitral valve, the pleomorphic variant, and the dimensions and extension. Complete surgical resection, including mitral valve replacement, is mandatory to improve symptoms and to obtain histologic information.

\section{Consent}

Written informed consent was obtained from the patient for publication of this case report and any accompanying images. A copy of the written consent is available for review by the Editor-in-Chief of this journal.

\section{Competing interests}

The authors declare that they have no competing interests.

\section{Authors' contributions}

EP performed the surgical procedure. FA preoperatively diagnosed the patient. MI performed the histopathological examination. AD performed the literature review. All authors were involved in drafting the manuscript or revising it critically for important intellectual content and have read and approved the final version. 


\section{Acknowledgement}

We would like to thank Mrs Enida Hoxha/Prifti for her support regarding the linguistic editing of the manuscript.

\section{Author details}

'Division of Cardiac Surgery, University Hospital Center of Tirana, Tirana, Albania. ${ }^{2}$ Division of Cardiology, Regional Hospital of Gjakovo, Gjakovo, Kosovo. ${ }^{3}$ Division of Heart Disease, Gjakovo Hospital, Rr. Prizren, Gjakove, Kosove.

Received: 2 May 2015 Accepted: 7 October 2015

Published online: 31 October 2015

\section{References}

1. O'Brien JE, Stout AP. Malignant fibrous xanthomas. Cancer. 1964;17:1155-445.

2. Shah AA, Churg A, Sbarbaro JA, Sheppard JM, Lamberti J. Malignant fibrous histiocytoma of the heart presenting as an atrial myxoma. Cancer. 1978:42:2466-71.

3. Okamoto K, Kato S, Katsuki S, Wada Y, Toyozumi Y, Morimatsu M, et al. Malignant fibrous histiocytoma of the heart: case report and review of 46 cases in the literature. Intern Med. 2001:40:1222-6.

4. Bandyopadhyay S, Banerjee S, Paul A, Das RK. Primary malignant fibrous histiocytoma involving the left pulmonary vein presenting as a left atrial tumor. Ann Card Anaesth. 2013;16(4):293-5.

5. Milicic D, Juretic A, Bulum J, Saric N, Bisof V, Jelic I, et al. Primary malignant fibrous histiocytoma of the heart with skeletal muscles metastases. J Card Surg. 2007;22(6):513-6.

6. Dorobantu M, Fruntelata A, Constantinescu D, Racoveanu I, Ardeleanu C, Tatu-Chitoiu G, et al. Primary left heart malignant fibrous histiocytoma. Eur J Echocardiogr. 2005;6(3):225-7.

7. Auer J, Berent R, Gurtner F. "Malignant" mitral stenosis. J Cardiothorac Surg. 2012;8:7-19.

8. Soo WM, Pang YH, Poh KK. Lethal mass mimicking myxoma in the heart. Asian Cardiovasc Thorac Ann. 2014;22(8):962-4.

9. Fontana A, Sciuchetti JF, Boffi L, Colagrande L, Trocino G. Unusual localization of a malignant fibrous histiocytoma on the mitral valve. Eur J Echocardiogr. 2010;11(1):77-9.

10. Wang CH, Yu HY, Chi NS, Chen YS, Lee KK, Cheng YJ, et al. Complete excision of primary cardiac malignant fibrous histiocytoma involving the left atrial free wall and mitral annulus by modified autotransplantation. J Thorac Cardiovasc Surg. 2006;131(3):731-3.

11. Sasaki K, Tanaka S, Ikeshita M, Shoji T, Fujita Y, Katagiri K. Malignant fibrous histiocytoma of the heart-a case report and review of the literature. Nihon Kyobu Geka Gakkai Zasshi. 1991;39(12):2240-5.

12. Baba T, Tanemoto K, Kuinose M, Kanaoka Y, Murakami T. A case of primary cardiac malignant fibrous histiocytoma. Kyobu Geka. 1998;51(10):849-52.

13. Fukumitsu T, Tsunekawa A, Watanabe M, Iwase M, Takeuchi E, Abe T. Primary malignant fibrous histiocytoma of the left atrium with acute mitral regurgitation. Am Heart J. 1988;115(3):691-3.

14. Schena S, Caniglia A, Agnino A, Caruso G, Ferlan G. Survival following treatment of a cardiac malignant fibrous histiocytoma. Chest. 2000;118:271-3.

\section{Submit your next manuscript to BioMed Central and take full advantage of:}

- Convenient online submission

- Thorough peer review

- No space constraints or color figure charges

- Immediate publication on acceptance

- Inclusion in PubMed, CAS, Scopus and Google Scholar

- Research which is freely available for redistribution 